\title{
Telomere Length is Not a Main factor for the Development of Islet Autoimmunity and Type 1 Diabetes in the TEDDY Study
}

Hemang Parikh ( $\sim$ parikhhemangm@gmail.com )

University of South Florida

Carina Törn

Lund University

Xiang Liu

University of South Florida

Suna Onengut-Gumuscu

University of Virginia

Kevin Counts

University of South Florida

Jose Moreno

University of South Florida

Cassandra Remedios

University of South Florida

Wei-Min Chen

University of Virginia

Jonathon LeFaive

University of Michigan-Ann Arbor

Martha Butterworth

University of South Florida

Beena Akolkar

National Institute of Diabetes and Digestive and Kidney Diseases

Jeffrey Krischer

University of South Florida

Åke Lernmark

Lund University

Marian Rewers

University of Colorado System

Jin-Xiong She

Augusta University

Jorma Toppari 
Turku University Hospital

\section{Anette-Gabriele Ziegler}

Helmholtz Zentrum München

\section{Aakrosh Ratan}

University of Virginia

Albert Smith

University of Michigan-Ann Arbor

William Hagopian

Pacific Northwest Diabetes Research Institute

\section{Stephen Rich}

University of Virginia

\section{Research Article}

Keywords: Telomere length, Computel, Islet autoimmunity, Type 1 diabetes

Posted Date: November 17th, 2021

DOI: https://doi.org/10.21203/rs.3.rs-1018251/v1

License: (1) (1) This work is licensed under a Creative Commons Attribution 4.0 International License. Read Full License 


\section{Abstract}

The Environmental Determinants of Diabetes in the Young (TEDDY) study enrolled 8,676 children, 3-4 months of age, born with HLA-susceptibility genotypes for islet autoimmunity (IA) and type 1 diabetes (T1D). Whole-genome sequencing (WGS) was performed in 1,119 children in a nested case-control study design. Telomere length was estimated from WGS data using five tools: Computel, Telseq, Telomerecat, qMotif and Motif_counter. The estimated median telomere length was $5.10 \mathrm{~kb}$ (IQR: $4.52 \mathrm{~kb}-5.68 \mathrm{~kb}$ ) using Computel. The age when the blood sample was drawn had a significant negative correlation with telomere length $(P=0.003)$. European children, particularly those from Finland $(P=0.041)$ and from Sweden $(P=0.001)$, had shorter telomeres than children from the U.S.A. Paternal age $(P=0.019)$ was positively associated with telomere length. First degree relative status, presence of gestational diabetes in the mother, and maternal age did not have a significant impact on estimated telomere length. HLA-DR4/4 or HLA-DR4/X children had significantly longer telomeres compared to children with HLA-DR3/3 or HLADR3/9 haplogenotypes $(P=0.008)$. Estimated telomere length was not significantly different with respect to any IA $(P=0.377)$, IAA-first $(P=0.248)$, GADA-first $(P=0.248)$ or T1D $(P=0.861)$.

These results suggest that telomere length has no major impact on the risk for IA, the first step to develop T1D. Nevertheless, telomere length was shorter in the T1D high prevalence populations, Finland and Sweden.

\section{Introduction}

Telomeres consist of repetitive nucleoprotein tracts of sequence (TTAGGG)n that cap the ends of linear chromosomes. Telomeres prevent the loss of coding sequences and hinder major chromosomal rearrangements ${ }^{1}$. Each cell division causes progressive attrition (around 50-200 bp of the telomeres) and is part of determining the lifespan of cells ${ }^{2}$. Rapidly dividing cell types are those within the immune system that proliferate not only to self-renew but also to perform their biological function.

Telomere length is, in part, inherited with a stronger influence on variation in telomere length from the father, defined by the stronger correlation in telomere length between father-son and father-daughter ${ }^{3,4}$. In addition, paternal age is positively correlated with longer telomeres ${ }^{5}$. At the population level, there is no difference in telomere length at birth between boys and girls ${ }^{6}$; however, in young children ${ }^{7}$ and young adults (around 30 years), telomeres are longer in women than in men, although the attrition rate is higher in women ${ }^{8}$. The preservation of telomeres in women may, therefore, occur during the first two decades of life ${ }^{9}$.

In healthy subjects, HLA-DRB $1 * 04$ alleles have been shown to be associated with shorter telomeres in $\mathrm{CD}^{+}{ }^{+}$T-cells, although the difference in telomere length between HLA-DR4 ${ }^{+}$and HLA-DR4- subjects was not identified at birth. Thus, during the first 20 years of life, telomere attrition may be accelerated in subjects with HLA-DR4 ${ }^{+10}$. 
Several mechanisms may contribute to the attrition of telomeres, but there is only one known mechanism in healthy individuals, beyond embryogenesis, that counteracts the shortening of telomeres. Some cell types, such as lymphocytes, are capable of activating telomerase, an enzyme that can elongate telomere sequences and thereby modulate cellular lifespan. Certain hormones can also stimulate telomerases. For example, estrogens have been shown to activate telomerase activity ${ }^{11}$ while cortisol can decrease telomerase activity. In vitro experiments have shown that high concentrations of hydrocortisone (comparable to cortisol levels that may occur in vivo due to stress) can reduce telomerase activity by up to $50 \%{ }^{12}$. Women with gestational diabetes (GDM), a condition of altered glucose metabolism and hormonal patterns during pregnancy, is associated with shorter telomeres in girls born to GDM mothers, but not in boys ${ }^{13}$.

Telomere length exhibits disease-specific patterns in different autoimmune diseases ${ }^{2,14,15}$. Shorter telomere length in leukocytes has been reported in subjects with type 1 diabetes (T1D) compared to controls, although shorter telomere length was not associated with the duration of T1D ${ }^{16}$. In T1D, allcause mortality is also associated with a shorter telomere length ${ }^{17}$.

Here, we utilize the TEDDY nested case-control (NCC) cohort to estimate telomere length from wholegenome sequencing (WGS) data. Analyses of telomere length were performed to find out if there was a relation to the development of islet autoimmunity (IA), type 1 diabetes (T1D), or both, as well as its association with HLA-DR-DQ haplogenotypes. Primary end-points in TEDDY included any IA, micro insulin autoantibody (mIAA)-first, the 65kDa glutamate decarboxylase autoantibody (GADA)-first, or T1D.

\section{Methods}

\section{Study design}

Screening of 421,047 newborn children for high-risk genotypes was performed at six clinical sites, three in Europe (Finland, Germany and Sweden) and three in the U.S.A. (Colorado (CO), Georgia (GA) and Washington (WA)) starting on 1 September 2004 and ending on 28 February 2010 as previously reported 18. The high-risk human leukocyte antigen (HLA)-haplogenotypes were as follows: HLA-DR3/4, HLADR4/4, HLA-DR4/8 or HLA-DR3/3 (Supplementary Table S1). HLA-DRB1*04 subtyping was performed to exclude those from the general population with HLA-DRB1*04:03. HLA-DQB1*03:04 also qualified for inclusion into the TEDDY study. HLA subtyping was not performed, so there was an inability to distinguish between HLA-DQB1*02 and HLA-DQA1*03 subtypes. In the HLA-DQB1*05:01 subtype, only HLA-DRB $1 * 01$ was included, while HLA-DRB1*10 was excluded.

The first blood sample for HLA-screening was obtained either as cord blood in the maternity clinic or as a dry blood spot (DBS) on day three or four. If the child was eligible for TEDDY, the family was contacted by a study nurse and invited to participate in the 15-year follow-up study with blood sampling for determining islet autoantibody (GADA, islet antigen-2 antibody (IA-2A) and mIAA) status every three months between 3 and 48 months of child's age and every six months thereafter (while a child with islet 
autoantibody positivity remained on the three months visit schedule). HLA-haplogenotypes were confirmed in a second blood sample at the 9-month visit. For the present study, blood samples were taken at the median age of 0.82 years (interquartile range (IQR): $0.75-5.43$ years) for extraction of DNA and subsequent sequencing.

All procedures performed in studies involving human participants were in accordance with the ethical standards of the institutional and/or national research committees and with the 1964 Helsinki declaration and its later amendments or comparable ethical standards. All procedures were approved by the ethics committees/institutional review boards including Colorado Multiple Institutional Review Board (04-0361); Medical College of Georgia Human Assurance Committee (2004-2010)/Georgia Health Sciences University Human Assurance Committee (2011-2012)/Georgia Regents University Institutional Review Board (2013-2017)/Augusta University Institutional Review Board (2017-present) (HAC 0405380); University of Florida Health Center Institutional Review Board (IRB201600277); Washington State Institutional Review Board (2004-2012)/Western Institutional Review Board (2013-present) (20130211); Ethics Committee of the Hospital District of Southwest Finland (Dnro168/2004); Bayerischen Landesärztekammer (Bavarian Medical Association) Ethics Committee (04089); and Regional Ethics Board in Lund, Section 2 (2004-2012)/Lund University Committee for Continuing Ethical Review (2013-present) (217/2004). In addition, TEDDY is monitored by an external evaluation committee formed by the National Institutes of Health, Bethesda, MD, U.S.A. Informed consent was obtained for all individual participants included in the study from a parent or primary caretaker, separately for genetic screening and participation in the prospective follow-up and WGS.

\section{Study outcomes: IA and T1D}

The primary outcome of the TEDDY study is the development of persistent confirmed IA assessed every three months. In the U.S.A., all sera were assayed at the Barbara Davis Center for Childhood Diabetes at the University of Colorado, Denver, CO; in Europe all sera were assayed at the University of Bristol, Bristol, U.K. GADA, IA-2A and mIAA were all measured using radio-binding assays ${ }^{19-21}$. IA was confirmed if an autoantibody was identified in a sample at both Reference Laboratories. All samples positive for IA and $5 \%$ of negative samples were re-tested for confirmation in both Reference Laboratories.

Persistent autoimmunity was defined by the presence of confirmed IA (GADA, IA-2A or mIAA) on two or more consecutive visits. The date of persistent confirmed IA was defined as the first blood draw date at which the child was found positive for at least one islet autoantibody. As children may be born with maternal IA, positive results due to maternal transmission via placenta were excluded when defining the child's IA status. If the child was IA-positive at 3 or 6 months of age, the IA status of the mother was assessed at the 9-month visit in order to distinguish maternal autoantibody from IA in the child. If maternal autoantibody was present, the child was not considered persistently IA-positive unless the child had a negative sample prior to the first positive sample.

The threshold for positivity differed by the laboratory. Positivity was defined by Bristol (GADA $\geq 33$ DK$\mathrm{U} / \mathrm{mL}, \mathrm{IA}-2 \mathrm{~A} \geq 5 \mathrm{DK}-\mathrm{U} / \mathrm{mL}$ and $\mathrm{mIAA}>0.95$ Local Units) and Denver (GADA > $20 \mathrm{DK}-\mathrm{U} / \mathrm{mL}, \mathrm{IA}-2 \mathrm{~A}>5 \mathrm{DK}-$ 
$\mathrm{U} / \mathrm{mL}$ and $\mathrm{mIAA}>0.010$ index). Both Reference Laboratories have shown high sensitivity and specificity as well as concordance for defining the presence of confirmed IA ${ }^{10,21}$.

\section{HLA typing}

HLA genotype screening was performed using either a dried blood spot (DBS) punch or a small volume blood specimen format ${ }^{18,22}$. After polymerase chain reaction (PCR) amplification of exon 2 of the HLA class II genes (DRB1, DQA1 or DQB1), alleles were identified either by direct sequencing, oligonucleotide probe hybridization or other genotyping techniques. Typing to certify specific HLA-DR-DQ haplogenotypes was determined for each clinical center. Confirmation of the HLA haplogenotypes was performed by the central HLA Reference Laboratory at Roche Molecular Systems, Oakland, CA on the eligible subjects using the 9-month sample.

\section{Estimation of telomere length from the WGS data}

WGS was performed on the subjects in the nested case-control (NCC) study for IA and T1D. In the NCC study, the "cases" were those subjects that had developed any IA or T1D as of 30 June 2018 and "controls" were randomly selected from those autoantibody-negative children in the index case's risk set, matched for sex, clinical site and first-degree relative (FDR) status ${ }^{23}$. Matching was performed in a 1:1 format (one control was matched to each case).

Extraction of DNA was performed by the Center for Public Health Genomics at the University of Virginia, VA, U.S.A. The WGS data were generated on the Illumina HiSeq X Series platform with paired-end $2 \times 150$ bp reads with targeted 30x coverage by Macrogen, Inc, Rockville, MD, U.S.A. FastQC, MultiQC ${ }^{24}$ and FastQ Screen ${ }^{25}$ were used to identify low-quality sequences and contaminants in the raw sequence data. The raw sequences were aligned to the Genome Reference Consortium Build 37 (GRCh37d5) for qMotif and to the Genome Reference Consortium Build 38 (GRCh38DH) for Computel, Telseq, Telomerecat and Motif_counter. Post-alignment processing was performed by utilizing the University of Michigan's (UM) Docker-alignment pipeline (https://github.com/statgen/docker-alignment) based on Burrows-Wheeler Aligner (BWA) ${ }^{26}$. Variant concordance analysis was performed by comparing the variants identified from the WGS data to the T1DExomeChip array (Illumina, CA) data to determine mislabeled, swapped or contaminated samples.

Estimation of the telomere length from the WGS data was performed using five different tools: Computel, Telseq, Telomerecat, qMotif and Motif_counter (Supplementary methods). Pairwise Spearman correlation analysis was used to compare performance of these tools against each other. All subsequent statistical analysis on telomere length was conducted using estimates from Computel which is based on aligning raw sequence data in FASTQ format to a telomeric reference sequence.

\section{SNPs associated with the telomere length}

Single nucleotide polymorphisms (SNPs) were genotyped by the Center for Public Health Genomics at the University of Virginia, VA, U.S.A., using the T1DExomeChip array on the full TEDDY cohort $(n=8,093)$ 
(Figure 1). The T1DExomeChip is a custom genotyping array with more than 90,000 custom content SNPs added to the Infinium ${ }^{\circledR}$ CoreExome-24 v.1.1 BeadChip (Illumina, CA). Genotype calling and the following quality control steps were applied to the dataset: (1) individuals with low call rate $(<95 \%)$, or discordance with reported sex and prior genotyping were not considered in the analysis, (2) SNPs with low call rates ( $<95 \%$ ) were excluded, (3) SNPs from autosomal chromosomes with allele distributions strongly deviating from Hardy-Weinberg equilibrium in controls with the European ancestry $\left(P<10^{-6}\right)$ were discarded (except for chromosome 6 with the Hardy-Weinberg equilibrium in controls with the European ancestry $\left(P<10^{-20}\right)$ due to HLA eligibility requirements), (4) SNPs on chromosome X were excluded with heterozygosity $>1 \%$ in men, (5) SNPs on chromosome $Y$ were excluded with genotypes $>$ $2 \%$ in women, (6) Monomorphic SNPs were excluded, (7) SNPs were discarded for which the concordance rate among duplicates was $<99 \%$ and (8) SNPs with missingness difference between IA or T1D cases and controls subjects assessed by the Fisher's exact test $\left(P<10^{-4}\right)$. From literature, there were 835 SNPs associated with telomere length on the T1DExomeChip ${ }^{27-38}$.

\section{Statistical analyses}

Multiple linear regression analysis was performed to examine the association between the telomere length and other factors including HLA-categories (HLA-DR3/3 or HLA-DR3/9 (as reference), HLA-DR3/4 and HLA-DR4/4, HLA-DR4/X), sex, parental age, mother's gestational diabetes status, FDR status and the country of residence (as strata). The age at sample collection and the indicator of the long-distance protocol sample collection (yes vs. no) were also included in the model. Telomere length data were available on a total of 1,119 TEDDY children.

Primary analyses were focused on examining the association between telomere length with the risk of any IA and/or T1D. A conditional logistic regression model was used to consider the matching criteria in the NCC study for any IA or T1D, adjusting for the HLA-categories (HLA-DR3/3 or HLA-DR3/9 (as reference), HLA-DR3/4 and HLA-DR4/4, HLA-DR4/X). In the analysis, telomere length was age-adjusted by using the residuals from the linear regression of telomere length on the age at sample collection. Subgroup analyses were conducted with respect to the type of first appearing autoantibody (mIAA-first and GADA-first). The magnitude of the association was estimated by odds ratio (OR), with $95 \%$ confidence internal $(\mathrm{Cl})$.

Secondary analyses were performed to examine the effect of SNPs associated with telomere length on the risk of any IA, mIAA-first, GADA-first and T1D. A total of 244 SNPs with minor allele frequency (MAF) > 0.05 were included (Figure 1). Cox proportional hazard models were used to study the association between each SNP and the risk of any IA, mIAA-first, GADA-first and T1D, respectively, adjusting for sex, the country of residence (as strata), FDR, HLA-haplogenotype and accounting for population stratilcation (ancestral heterogeneity) (using the top three principal components estimated from the T1DExomeChip). Of the 8,676 enrolled children, 583 were excluded: indeterminate HLA haplogenotypes $(n=14), H L A$ ineligible $(n=112)$, and no SNP data available $(n=457)$. A total of 7,093 families had one child, 476 families had 2 children, and 16 families had 3 children who were included in the analysis. A robust 
variance estimate was used to account for the dependence within families in the Cox proportional hazard model ${ }^{39}$. The magnitude of each estimated association was defined by the Hazard ratio (HR), with $95 \%$ confidence intervals (Cls). To correct for multiple hypothesis testing, the false discovery rate adjusted $P$ values were calculated ${ }^{40}$. SNPs with nominal significance can be found in Supplementary Table S3 through S6.

Statistical analyses were performed using Statistical Analysis Software (Version 9.4, SAS Institute, Cary, North Carolina, U.S.A.) and R (Version 4.0.2). For the multiple linear regression analysis, estimates with $P$ $<0.05$ were considered as statistically significant.

\section{Results}

Telomere lengths from the WGS data were calculated from 1,119 TEDDY children (Figure 1). Descriptive data on the estimation of the telomere lengths is shown in Table 1. The estimated median telomere length was $5.10 \mathrm{~kb}$ (IQR: $4.52 \mathrm{~kb}-5.68 \mathrm{~kb}$ ) using Computel. The estimations of the telomere lengths were strongly correlated between Computel and Telseq $(r=0.97, P<0.001)$ as well as between Computel and Motif_counter $(r=0.93, P<0.001)$ (Supplemental Figure $S 1)$. 
Table 1

Length of telomeres $(\mathrm{kb})$ in study subjects by the status of islet autoimmunity $(\mathrm{IA})$ and type 1 diabetes (T1D) in the Environmental Determinants of Diabetes in the Young (TEDDY) study. Telomere length data were available on both case and matched control subjects in 389 IA pairs and 118 T1D pairs. Computel was used to estimate the telomere length from the whole-genome sequence (WGS) data.

\begin{tabular}{|lllllll|}
\hline Parameter & \multicolumn{2}{l}{ Islet autoimmunity (IA) } & \multicolumn{3}{l|}{ Type 1 diabetes (T1D) } \\
\hline & $\begin{array}{l}\text { Matched } \\
\text { pairs (\%) }\end{array}$ & $\begin{array}{l}\text { IA cases } \\
\text { (in kb) }\end{array}$ & $\begin{array}{l}\text { IA controls } \\
\text { (in kb) }\end{array}$ & $\begin{array}{l}\text { Matched } \\
\text { pairs (\%) }\end{array}$ & $\begin{array}{l}\text { T1D cases } \\
\text { (in kb) }\end{array}$ & $\begin{array}{l}\text { T1D } \\
\text { controls (in } \\
\text { kb) }\end{array}$ \\
\hline Country & & & & & & \\
\hline Finland & 63 & $5.07 \pm$ & $5.22 \pm$ & 23 & $5.09 \pm$ & $5.02 \pm 0.93$ \\
& $(16.20 \%)$ & 0.76 & 0.77 & $(19.49 \%)$ & 0.78 & \\
\hline Germany & $13(3.34 \%)$ & $5.13 \pm$ & $5.52 \pm$ & $5(4.24 \%)$ & $5.39 \pm$ & $5.40 \pm 0.27$ \\
& & 0.65 & 1.08 & & 0.96 & \\
Sweden & 182 & $5.13 \pm$ & $4.94 \pm$ & 49 & $5.22 \pm$ & $5.05 \pm 1.05$ \\
& $(46.78 \%)$ & 0.76 & 0.84 & $(41.53 \%)$ & 0.87 & \\
U.S.A. & 131 & $5.24 \pm$ & $5.35 \pm$ & 41 & $5.16 \pm$ & $5.09 \pm 0.84$ \\
& $(33.68 \%)$ & 0.78 & 0.84 & $(34.74 \%)$ & 0.73 & \\
\hline
\end{tabular}

Family

history of

T1D

\begin{tabular}{lllllll}
$\begin{array}{l}\text { First-degree } \\
\text { relative }\end{array}$ & 79 & $5.19 \pm$ & $5.19 \pm$ & 25 & $5.27 \pm$ & $5.07 \pm 0.81$ \\
General & $(20.31 \%)$ & 0.64 & 0.81 & $(21.19 \%)$ & 0.84 & \\
population & $(79.69 \%)$ & $0.79 \pm$ & $5.13 \pm$ & 93 & $5.16 \pm$ & $5.07 \pm 0.96$ \\
\hline
\end{tabular}

Sex

$\begin{array}{lllllll}\text { Female } & 169 & 5.23 \pm & 5.21 \pm & 52 & 5.28 \pm & 5.09 \pm 0.96 \\ & (43.44 \%) & 0.73 & 0.88 & (44.07 \%) & 0.75 & \\ \text { Male } & 220 & 5.10 \pm & 5.10 \pm & 66 & 5.10 \pm & 5.06 \pm 0.91 \\ & (56.56 \%) & 0.78 & 0.84 & (55.93 \%) & 0.84 & \end{array}$

Telomere length is presented as mean \pm standard deviation (S.D.) in kb.

\section{Association between telomere length and other phenotypes}

The age of the child when the sample was drawn for WGS had a significant impact on the length of telomeres. There was a significant negative association between age of the children and telomere length $(P=0.003)$ (Table 2). Children that carried HLA-DR4/4 or HLA-DR4/X had significantly longer telomeres compared to children with HLA-DR3/3 or HLA-DR3/9 haplogenotypes $(P=0.008)$. European children, particularly those from Finland $(P=0.041)$ and from Sweden $(P=0.001)$, had shorter telomeres than children from the U.S.A. Paternal age $(P=0.019)$ and the female sex of the child $(P=0.069$; although not 
significant) were positively associated with telomere length. FDR status, presence of GDM in the mother, and maternal age did not have a significant impact on estimated telomere length (Table 2).

Table 2

Association between telomere length and characteristics of the subjects (sex, high risk HLADR-DQ haplogenotypes, country, first-degree relative (FDR) with T1D, gestational diabetes in the mother and parental age). Telomere length data were available on a total of 1,119 TEDDY children. The age at sample collection and the indicator of the long-distance protocol sample collection (yes vs. no) were also included in the model.

\begin{tabular}{|c|c|c|c|}
\hline Parameter & Regression coefficient & Standard error & $P$-value \\
\hline Child's age (years) & -0.029 & 0.0097 & 0.003 \\
\hline \multicolumn{4}{|l|}{ Sex } \\
\hline Female & 0.083 & 0.0458 & 0.069 \\
\hline \multicolumn{4}{|l|}{ High risk HLA-DR-DQ haplogenotypes } \\
\hline HLA-DR3/3 or HLA-DR3/9 & Reference & Reference & Reference \\
\hline HLA-DR3/4 & 0.105 & 0.0647 & 0.106 \\
\hline HLA-DR4/4 or HLA-DR4/X & 0.179 & 0.0675 & 0.008 \\
\hline \multicolumn{4}{|l|}{ Country } \\
\hline U.S.A. & Reference & Reference & Reference \\
\hline Finland & -0.137 & 0.0671 & 0.041 \\
\hline Germany & -0.100 & 0.1279 & 0.432 \\
\hline Sweden & -0.191 & 0.0548 & 0.001 \\
\hline \multicolumn{4}{|l|}{ FDR with T1D } \\
\hline General population & Reference & Reference & Reference \\
\hline Father & 0.109 & 0.0771 & 0.157 \\
\hline Mother & -0.044 & 0.0985 & 0.653 \\
\hline Sibling & -0.054 & 0.1203 & 0.653 \\
\hline Gestational diabetes in the mother & 0.094 & 0.0943 & 0.317 \\
\hline \multicolumn{4}{|l|}{ Parental age } \\
\hline Maternal age & 0.002 & 0.0064 & 0.792 \\
\hline Paternal age & 0.013 & 0.0055 & 0.019 \\
\hline
\end{tabular}

\section{Association between telomere length and the risk of any IA, mIAA-first, GADA-first and T1D}


Estimated telomere length was not significantly different with respect to any IA (OR $=0.91,95 \% \mathrm{Cl}$ : 0.75 $1.12, P=0.377$ ), mIAA-first ( $\mathrm{OR}=1.23,95 \% \mathrm{Cl}: 0.87-1.73, P=0.248)$, GADA-first (OR $=0.83,95 \% \mathrm{Cl}: 0.60$ $1.14, P=0.248)$ or T1D $(\mathrm{OR}=1.03,95 \% \mathrm{Cl}: 0.75-1.42, P=0.861)$ (Table 3$)$. There was a significantly increased risk for children with HLA-DR3/4 (OR $=1.85,95 \% \mathrm{Cl}: 1.23-2.78, P=0.003)$ to develop IA regardless of type of islet autoantibody (any IA) as compared with those with HLA-DR3/3 or HLA-DR3/9 haplogenotypes. The risk to develop mIAA as the first autoantibody was significantly increased for children with HLA-DR3/4 (OR = 3.73, 95\% Cl: $1.70-8.17, P=0.001)$ and HLA-DR4/4 or HLA-DR4/X (OR = $2.59,95 \% \mathrm{Cl}: 1.16-5.78, P=0.020$ ) as compared to those with HLA-DR3/3 or HLA-DR3/9. The risks to develop GADA as the first autoantibody or T1D was not associated with any of the HLA-DR-DQ haplogenotypes (Table 3). 
Table 3

Association between age-adjusted telomere length in subjects by the status of islet autoimmunity (IA, GADA-first or mIAA-first) and T1D in relation to HLA haplogenotypes. Complete data were available on 389 nested case-control (NCC) pairs for IA and on 118 matched pairs for T1D.

\begin{tabular}{|c|c|c|}
\hline HLA status & Odds ratio $(95 \% \mathrm{Cl})$ & $P$-value \\
\hline \multicolumn{3}{|l|}{ Any IA ( $n=389$ NCC pairs) } \\
\hline Age-adjusted telomere length & $0.91(0.75-1.12)$ & 0.377 \\
\hline HLA-DR3/3 or HLA-DR3/9 & Reference & Reference \\
\hline HLA-DR3/4 & $1.85(1.23-2.78)$ & 0.003 \\
\hline HLA-DR4/4 or HLA-DR4/X & $1.30(0.84-2.02)$ & 0.236 \\
\hline \multicolumn{3}{|l|}{ mIAA-first ( $n=159$ NCC pairs) } \\
\hline Age-adjusted telomere length & $1.23(0.87-1.73)$ & 0.248 \\
\hline HLA-DR3/3 or HLA-DR3/9 & Reference & Reference \\
\hline HLA-DR3/4 & $3.73(1.70-8.17)$ & 0.001 \\
\hline HLA-DR4/4 or HLA-DR4/X & $2.59(1.16-5.78)$ & 0.020 \\
\hline \multicolumn{3}{|l|}{ GADA-first ( $n=158$ NCC pairs) } \\
\hline Age-adjusted telomere length & $0.83(0.60-1.14)$ & 0.248 \\
\hline HLA-DR3/3 or HLA-DR3/9 & Reference & Reference \\
\hline HLA-DR3/4 & $0.91(0.51-1.63)$ & 0.762 \\
\hline HLA-DR4/4 or HLA-DR4/X & $0.69(0.36-1.33)$ & 0.266 \\
\hline \multicolumn{3}{|l|}{ T1D (n = 118 NCC pairs) } \\
\hline Age-adjusted telomere length & $1.03(0.75-1.42)$ & 0.861 \\
\hline HLA-DR3/3 or HLA-DR3/9 (Reference) & Reference & Reference \\
\hline HLA-DR3/4 & $2.22(0.97-5.10)$ & 0.060 \\
\hline HLA-DR4/4 or HLA-DR4/X & $1.63(0.71-3.73)$ & 0.249 \\
\hline
\end{tabular}

\section{SNPs associated with telomere length and the risk of any IA, mIAA-first, GADA-first and T1D}

We selected 236 SNPs previously reported to be associated with telomere length (see Material and methods). A total of 31 SNPs in 13 regions were nominally associated with risk of developing any IA, mIAA-first, GADA-first or T1D; however, none of these associations withstood correction for multiple 
hypothesis testing (Supplementary Table S3 through S6). Six SNPs had nominally significant associations to more than one endpoint. rs11082257, an intronic SNP in PIK3C3 on chromosome 18q12.3, was associated with decreased risk for any IA (HR=0.88, 95\% Cl: $0.78-0.99, P=0.031)$, mIAAfirst (HR $=0.76,95 \% \mathrm{Cl}: 0.62-0.93, P=0.008)$ and T1D (HR=0.79, 95\% Cl: $0.65-0.97, P=0.022)$. rs3017077 in MRE11 on chromosome 11q21 was associated with decreased risk for any IA (HR $=0.90$, $95 \% \mathrm{Cl}: 0.81-1.00, P=0.044)$ and mIAA-first (HR $=0.83,95 \% \mathrm{Cl}: 0.69-0.99, P=0.038)$. rs75245322, an intronic SNP in RAD50 on chromosome 5q31.1, was associated with increased risk for any IA (HR $=1.21$, 95\% Cl: $1.02-1.44, P=0.030)$ and T1D (HR = 1.36, 95\% Cl: $1.05-1.76, P=0.018)$. rs7167216, a missense SNP in $B L M$ on chromosome 15q26.1, was associated with increased risk for GADA-first (HR $=1.32,95 \%$ $\mathrm{Cl}: 1.04-1.67, P=0.022)$ and T1D (HR=1.30, 95\% Cl: $1.02-1.66, P=0.038)$. rs1801516, a missense SNP in ATM on chromosome 11q22.3, was associated with increased risk for any IA (HR $=1.14,95 \% \mathrm{Cl}$ : 1.00 1.30, $P=0.045)$ and mIAA-first ( $\mathrm{HR}=1.24,95 \% \mathrm{Cl}: 1.01-1.53, P=0.041)$. rs4353905 on chromosome $4 q 25$ was associated with increased risk for any IA ( $H R=1.15,95 \% \mathrm{Cl}: 1.01-1.31, P=0.032)$ and mIAAfirst (HR=1.32, 95\% Cl: $1.08-1.62, P=0.006)$. All of the SNPs were located on the q-arms (long arms).

\section{Discussion}

The key finding in this study was that children from high incidence countries for T1D i.e., Finland and especially from Sweden had shorter telomeres in white blood cells as compared to children from other TEDDY sites (Germany and three sites in the U.S.A.). The age when the blood sample was drawn had a significant negative correlation with telomere length, consistent with previous reports ${ }^{41}$. Interestingly, children that carried HLA-DR4/4 or HLA-DR4/X had significantly longer telomeres compared to children with HLA-DR3/3 or HLA-DR3/9 haplogenotypes. Also, paternal age was significantly correlated with telomere length. Children with HLA-DR3/4 had a significantly increased risk to develop any IA as compared to children with HLA-DR3/3 or HLA-DR3/9 haplogenotypes. Moreover, there were significantly increased risks for children with HLA-DR3/4 and HLA-DR4/4 or HLA-DR4/X to develop mIAA as the first autoantibody as compared to those with HLA-DR3/3 or HLA-DR3/9 haplogenotypes. In contrast, GADA as the first autoantibody or T1D were not associated with any of the HLA-DR-DQ haplogenotypes.

To our knowledge, there has been no reports on the risk for IA, mIAA-first or GADA-first in relation to telomere length. The estimations of the telomeres were highly correlated between Computel, Telseq and Motif_counter with $\mathrm{R}^{2}$ values ranging from 0.85 to 0.94 (Supplemental Figure 1). The finding of similar telomere lengths in young boys and girls confirms a previous study of telomere length taken at birth ${ }^{6}$. There has been a previous report on shorter telomere length in children with T1D ${ }^{16}$, a finding that was not reproduced here. This discrepancy could be explained by the older age of the participants in the previous report (17 to 48 years), compared with the TEDDY study in which the oldest children with T1D were 14.16 years. We could not reproduce the findings of shorter telomeres in children born to mothers with GDM, although this could be due to the older age (9 to 16 years) in the earlier study ${ }^{13}$. In addition, we detected

a significant effect of paternal age but not maternal age on the length of telomeres ${ }^{5}$. The SNPs that had nominally significant association to our primary and secondary outcomes - any IA, mIAA-first, GADA-first 
or T1D - were mainly from genes coding for proteins involved in double DNA strand repair and maintenance of telomeres (MRE11 and RAD50) ${ }^{42}$, DNA replication and repair (BLM) ${ }^{43}$, genome stability $(A T M)^{44}$ or degradative endocytic trafficking (PIK3C3) ${ }^{45}$. The $B L M$ gene has gene ontology including nucleic acid binding and ATPase activity also involved in DNA replication and repair.

The major strength of the present study is the use of both IA and T1D as endpoints. Additional strengths of this study are the inclusion of unrelated subjects from both the general population and FDRs without islet autoantibodies or T1D from the same countries in the same age ranges as the subjects with outcome events. One limitation of the present study is the relatively low number of subjects with IA, particularly those with GADA-first or with T1D, raising concerns for statistical power of the association analysis. Nevertheless, the screening of more than 400,000 newborns in four different countries to identify those with an increased genetic risk for T1D, defined by high-risk HLA-DR-DQ haplogenotypes, followed with blood draws every three months for the first four years and every six months thereafter is a unique effort. The proportional hazard ratio identifies risk for time-to-event in this longitudinal study, thereby increasing the power compared with cross-sectional case-control study design.

We concluded that genes in the HLA-region particularly HLA-DR3/4 and HLA-DR4/4, HLA-DR4/X remain the most important genetic risk factors for IA, the first step towards T1D. Children with the high-risk genotypes for T1D from Finland and Sweden had shorter telomeres and telomere length is associated with HLA-DR4/4 or HLA-DR4/X haplogenotype as compared to HLA-DR3/3 or HLA-DR3/9. Moreover, paternal age was positively associated with telomere length.

\section{Declarations}

\section{Competing interests}

The authors declare no competing interests associated with this study.

\section{Availability of data and material}

The datasets generated and analyzed during the current study will be made available in the NIDDK Central Repository at https://repository.niddk.nih.gov/studies/teddy/. The TEDDY Whole Genome Sequencing (WGS) data that support the findings of this study have been deposited in NCBI's database of Genotypes and Phenotypes (dbGaP) with the primary accession code phs001442.v3.p2.

\section{Authors contributions}

C.T., X.L., B.A., J.P.K., Å.L., M.R., J-X.S., J.T., A-G.Z., W.A.H., S.S.R., H.M.P. provided conception and design of the study. B.A., J.P.K., Å.L., M.R., J-X.S., J.T., A-G.Z., W.A.H. participated in patient recruitment and diagnosis, sample collection, and generation of the TEDDY metadata. S.O-G., S.S.R. processed the samples and generated the whole-genome sequencing (WGS) data. S.O-G., W-M.C., S.S.R. processed the samples and generated the TEDDY-T1DExomeChip data. S.O-G., K.M.C., J.L.M., C.L.R., J.L., A.V.S., H.M.P. 
performed QC and alignments of the WGS data. X.L., S.O-G., K.M.C., J.L.M., W-M.C., M.D.B., H.M.P. performed QC and analysis of the TEDDY-T1DExomeChip data. C.T., X.L., K.M.C., J.L.M., C.L.R., J.L., M.D.B., A.R., A.V.S., S.S.R., H.M.P. performed analysis to estimate the telomere length from the WGS data. X.L., M.D.B., H.M.P. conducted statistical analyses and researched the telomere length and TEDDYT1DExomeChip data. C.T., X.L., H.M.P. wrote the manuscript. All authors contributed to critical revisions and approved the final manuscript.

\section{Code availability}

The code is available on GitHub page: https://github.com/USF-HII/pub-teddy-human-wgs-telomere.

\section{Funding}

The TEDDY Study is funded by U01 DK63829, U01 DK63861, U01 DK63821, U01 DK63865, U01 DK63863, U01 DK63836, U01 DK63790, UC4 DK63829, UC4 DK63861, UC4 DK63821, UC4 DK63865, UC4 DK63863, UC4 DK63836, UC4 DK95300, UC4 DK100238, UC4 DK106955, UC4 DK112243, UC4 DK117483, U01 DK124166, U01 DK128847, and Contract No. HHSN267200700014C from the National Institute of Diabetes and Digestive and Kidney Diseases (NIDDK), National Institute of Allergy and Infectious Diseases (NIAID), Eunice Kennedy Shriver National Institute of Child Health and Human Development (NICHD), National Institute of Environmental Health Sciences (NIEHS), Centers for Disease Control and Prevention (CDC), and JDRF. This work supported in part by the NIH/NCATS Clinical and Translational Science Awards to the University of Florida (UL1 TR000064) and the University of Colorado (UL1 TR001082) as well as by the Academy of Finland (grant 276475).

\section{References}

1. Blackburn, E. H. \& TelomeresTrends Biochem Sci16,378-381, doi:10.1016/0968-0004(91)90155-o (1991)

2. Goronzy, J. J., Fujii, H. \& Weyand, C. M. Telomeres, immune aging and autoimmunity. Exp Gerontol, 41, 246-251 https://doi.org/doi:10.1016/j.exger.2005.12.002 (2006).

3. Gardner, M. et al. Gender and telomere length: systematic review and meta-analysis. Exp Gerontol, 51, 15-27 https://doi.org/doi:10.1016/j.exger.2013.12.004 (2014).

4. Nordfjall, K., Larefalk, A., Lindgren, P., Holmberg, D. \& Roos, G. Telomere length and heredity: Indications of paternal inheritance. Proc Natl Acad Sci U S A, 102, 16374-16378 https://doi.org/doi:10.1073/pnas.0501724102 (2005).

5. Broer, L. et al. Meta-analysis of telomere length in 19,713 subjects reveals high heritability, stronger maternal inheritance and a paternal age effect. Eur J Hum Genet, 21, 1163-1168 https://doi.org/doi:10.1038/ejhg.2012.303 (2013).

6. Okuda, K. et al. Telomere length in the newborn. Pediatr Res, 52, 377-381 https://doi.org/doi:10.1203/00006450-200209000-00012 (2002). 
7. Ly, K. et al. Telomere length in early childhood is associated with sex and ethnicity. Sci Rep, 9, 10359 https://doi.org/doi:10.1038/s41598-019-46338-x (2019).

8. Kark, J. D., Goldberger, N., Kimura, M., Sinnreich, R. \& Aviv, A. Energy intake and leukocyte telomere length in young adults. Am J Clin Nutr, 95, 479-487 https://doi.org/doi:10.3945/ajcn.111.024521 (2012).

9. Dalgard, C. et al. Leukocyte telomere length dynamics in women and men: menopause vs age effects. Int J Epidemiol, 44, 1688-1695 https://doi.org/doi:10.1093/ije/dyv165 (2015).

10. Schonland, S. O. et al. Premature telomeric loss in rheumatoid arthritis is genetically determined and involves both myeloid and lymphoid cell lineages. Proc Natl Acad Sci U S A, 100, 13471-13476 https://doi.org/doi:10.1073/pnas.2233561100 (2003).

11. Kyo, S. et al. Estrogen activates telomerase. Cancer Res, 59, 5917-5921 (1999).

12. Choi, J., Fauce, S. R. \& Effros, R. B. Reduced telomerase activity in human T lymphocytes exposed to cortisol. Brain Behav Immun, 22, 600-605 https://doi.org/doi:10.1016/j.bbi.2007.12.004 (2008).

13. Hjort, L. et al. Telomere length is reduced in 9- to 16-year-old girls exposed to gestational diabetes in utero., 61, 870-880 https://doi.org/doi:10.1007/s00125-018-4549-7 (2018).

14. Andrews, N. P., Fujii, H., Goronzy, J. J. \& Weyand, C. M. Telomeres and immunological diseases of aging. Gerontology, 56, 390-403 https://doi.org/doi:10.1159/000268620 (2010).

15. Hohensinner, P. J., Goronzy, J. J. \& Weyand, C. M. Telomere dysfunction, autoimmunity and aging. Aging Dis, 2, 524-537 (2011).

16. Jeanclos, E. et al. Shortened telomere length in white blood cells of patients with IDDM., 47, 482-486 https://doi.org/doi:10.2337/diabetes.47.3.482 (1998).

17. Astrup, A. S. et al. Telomere length predicts all-cause mortality in patients with type 1 diabetes., 53, 45-48 https://doi.org/doi:10.1007/s00125-009-1542-1 (2010).

18. Hagopian, W. A. et al. The Environmental Determinants of Diabetes in the Young (TEDDY): genetic criteria and international diabetes risk screening of 421000 infants. Pediatr Diabetes, 12, 733-743 https://doi.org/doi:10.1111/j.1399-5448.2011.00774.x (2011).

19. Babaya, N. et al. Comparison of insulin autoantibody: polyethylene glycol and micro-IAA 1-day and 7day assays. Diabetes Metab Res Rev, 25, 665-670 https://doi.org/doi:10.1002/dmrr.1014 (2009).

20. Bonifacio, E. et al. Harmonization of glutamic acid decarboxylase and islet antigen-2 autoantibody assays for national institute of diabetes and digestive and kidney diseases consortia. J Clin Endocrinol Metab, 95, 3360-3367 https://doi.org/doi:10.1210/jc.2010-0293 (2010).

21. Torn, C. et al. Diabetes Antibody Standardization Program: evaluation of assays for autoantibodies to glutamic acid decarboxylase and islet antigen-2., 51, 846-852 https://doi.org/doi:10.1007/s00125-008-0967-2 (2008).

22. Dantonio, P. et al. Proficiency testing of human leukocyte antigen-DR and human leukocyte antigenDQ genetic risk assessment for type 1 diabetes using dried blood spots. J Diabetes Sci Technol, 4, 929-941 https://doi.org/doi:10.1177/193229681000400424 (2010). 
23. Lee, H. S. et al. Biomarker discovery study design for type 1 diabetes in The Environmental Determinants of Diabetes in the Young (TEDDY) study. Diabetes Metab Res Rev, 30, 424-434 https://doi.org/doi:10.1002/dmrr.2510 (2014).

24. Ewels, P., Magnusson, M., Lundin, S. \& Kaller, M. MultiQC: summarize analysis results for multiple tools and samples in a single report., 32, 3047-3048 https://doi.org/doi:10.1093/bioinformatics/btw354 (2016).

25. Wingett, S. W., Andrews, S. \& FastQ Screen A tool for multi-genome mapping and quality control. F1000Res, 7, 1338 https://doi.org/doi:10.12688/f1000research.15931.2 (2018).

26. Li, H. \& Durbin, R. Fast and accurate short read alignment with Burrows-Wheeler transform., 25, 1754-1760 https://doi.org/doi:10.1093/bioinformatics/btp324 (2009).

27. Bodelon, C. et al. On the interplay of telomeres, nevi and the risk of melanoma. PLoS One, 7, e52466 https://doi.org/doi:10.1371/journal.pone.0052466 (2012).

28. Codd, V. et al. Common variants near TERC are associated with mean telomere length. Nat Genet, 42 , 197-199 https://doi.org/doi:10.1038/ng.532 (2010).

29. Codd, V. et al. Identification of seven loci affecting mean telomere length and their association with disease. Nat Genet, 45, 422-427 https://doi.org/doi:10.1038/ng.2528 (2013). 427e421-422,

30. Delgado, D. A. et al. The contribution of parent-to-offspring transmission of telomeres to the heritability of telomere length in humans. Hum Genet, 138, 49-60 https://doi.org/doi:10.1007/s00439-018-1964-2 (2019).

31. Dorajoo, R. et al. Loci for human leukocyte telomere length in the Singaporean Chinese population and trans-ethnic genetic studies. Nat Commun, 10, 2491 https://doi.org/doi:10.1038/s41467-01910443-2 (2019).

32. Fang, J. et al. Functional characterization of a multi-cancer risk locus on chr5p15.33 reveals regulation of TERT by ZNF148. Nat Commun, 8, 15034 https://doi.org/doi:10.1038/ncomms15034 (2017).

33. Kuo, C. L., Pilling, L. C., Kuchel, G. A., Ferrucci, L. \& Melzer, D. Telomere length and aging-related outcomes in humans: A Mendelian randomization study in 261,000 older participants., 18, e13017 https://doi.org/doi:10.1111/acel.13017 (2019).

34. Lee, J. H. et al. Genome wide association and linkage analyses identified three loci-4q25, 17q23.2, and 10q11.21-associated with variation in leukocyte telomere length: the Long Life Family Study. Front Genet, 4, 310 https://doi.org/doi:10.3389/fgene.2013.00310 (2013).

35. Liu, Y. et al. A genome-wide association study identifies a locus on TERT for mean telomere length in Han Chinese. PLoS One, 9, e85043 https://doi.org/doi:10.1371/journal.pone.0085043 (2014).

36. Mangino, M. et al. DCAF4, a novel gene associated with leucocyte telomere length. J Med Genet, 52, 157-162 https://doi.org/doi:10.1136/jmedgenet-2014-102681 (2015).

37. Mangino, M. et al. A genome-wide association study identifies a novel locus on chromosome 18q12.2 influencing white cell telomere length. J Med Genet, 46, 451-454 https://doi.org/doi:10.1136/jmg.2008.064956 (2009). 
38. Walsh, K. M. et al. Variants near TERT and TERC influencing telomere length are associated with high-grade glioma risk. Nat Genet, 46, 731-735 https://doi.org/doi:10.1038/ng.3004 (2014).

39. Wei, L. J., Lin, D. Y. \& Weissfeld, L. Regression-Analysis of Multivariate Incomplete Failure Time Data by Modeling Marginal Distributions. J Am Stat Assoc, 84, 1065-1073 https://doi.org/doi:Doi 10.2307/2290084 (1989).

40. Benjamini, Y. \& Hochberg, Y. Controlling the False Discovery Rate - a Practical and Powerful Approach to Multiple Testing. J R Stat Soc B, 57, 289-300 https://doi.org/doi:DOI 10.1111/j.25176161.1995.tb02031.x (1995).

41. Turner, K. J., Vasu, V. \& Griffin, D. K. Telomere Biology and Human Phenotype., 8, https://doi.org/doi:10.3390/cells8010073 (2019).

42. Paull, T. T. \& Gellert, M. The $3^{\prime}$ to $5^{\prime}$ exonuclease activity of Mre 11 facilitates repair of DNA doublestrand breaks. Mol Cell, 1, 969-979 https://doi.org/doi:10.1016/s1097-2765(00)80097-0 (1998).

43. Manthei, K. A. \& Keck, J. L. The BLM dissolvasome in DNA replication and repair. Cell Mol Life Sci, 70, 4067-4084 https://doi.org/doi:10.1007/s00018-013-1325-1 (2013).

44. Lavin, M. F. et al. ATM signaling and genomic stability in response to DNA damage. Mutat Res, 569, 123-132 https://doi.org/doi:10.1016/j.mrfmmm.2004.04.020 (2005).

45. Munson, M. J., Ganley, I. G. \& MTOR PIK3C3, and autophagy: Signaling the beginning from the end. Autophagy, 11, 2375-2376 https://doi.org/doi:10.1080/15548627.2015.1106668 (2015).

\section{Figures}




\section{Figure 1}

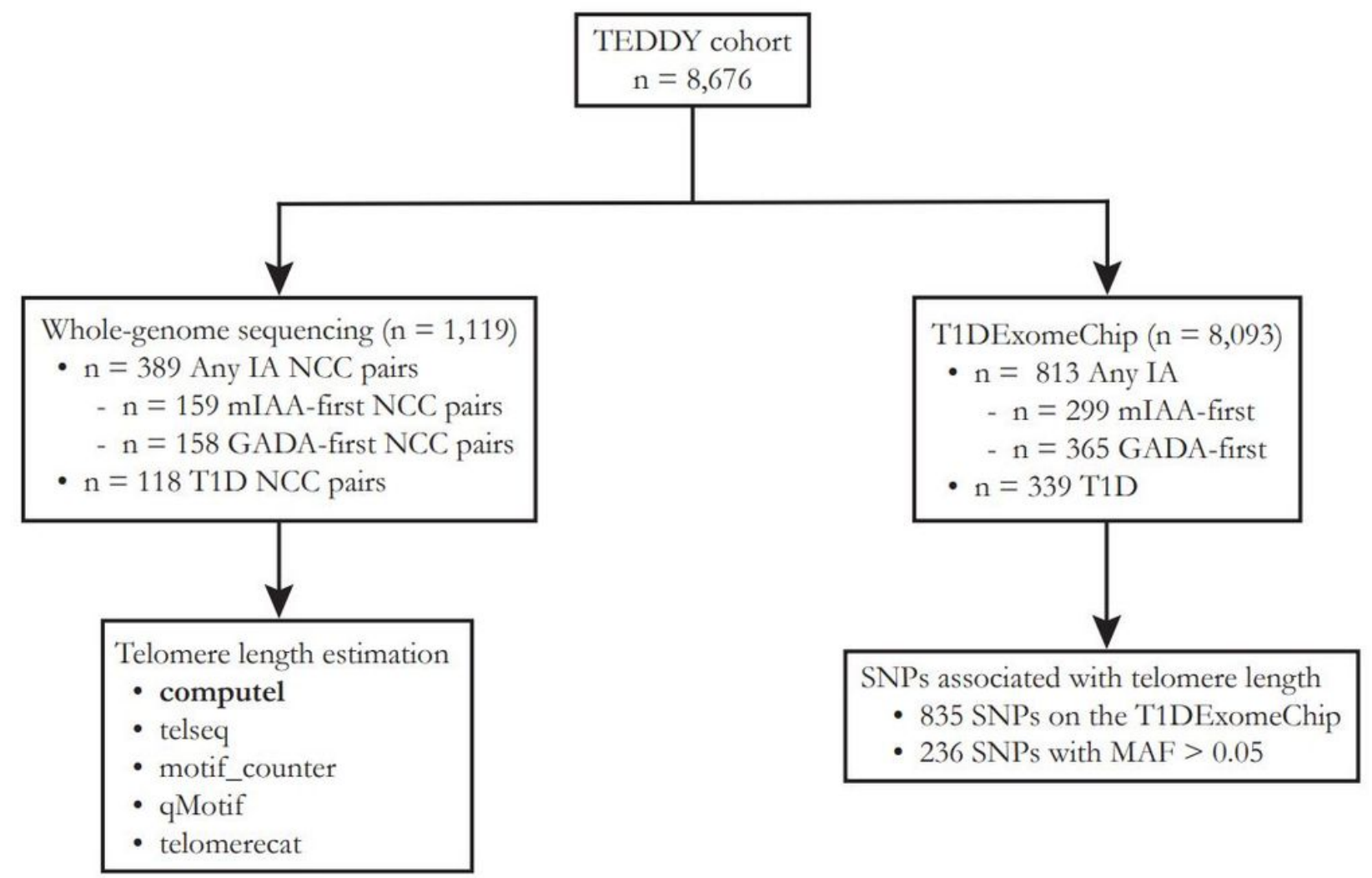

\section{Figure 1}

Whole-genome sequencing (WGS) was performed on the subjects in the nested case-control (NCC) study for islet autoimmunity (IA) and type 1 diabetes (T1D). In the NCC study, the "cases" were those subjects that had developed any IA $(n=389)$ or T1D $(n=118)$ and "controls" were randomly selected from those autoantibody-negative children in the index case's risk set, matched for sex, clinical site and first-degree relative (FDR) status. Telomere length estimation was performed using five different tools. Single nucleotide polymorphisms (SNPs) were genotyped using the T1DExomeChip array. A total of 835 SNPs associated with telomere length were available on the T1DExomeChip and 236 of those had a minor allele frequency $(\mathrm{MAF})>0.05$. SNP analysis was performed on 8,093 TEDDY children.

\section{Supplementary Files}

This is a list of supplementary files associated with this preprint. Click to download.

- TornMP140SupplementaryMethods20211025.docx

- SuppFigure120211106.pdf

- TEDDYStudyGroup20211025.docx 